\title{
Low-frequency divergence of the dielectric constant in metal-insulator nanocomposites with tunneling
}

\author{
A. B. Pakhomov, * S. K. Wong, X. Yan, and X. X. Zhang \\ Department of Physics, The Hong Kong University of Science and Technology, Clear Water Bay, Kowloon, Hong Kong, China
}

(Received 20 March 1998; revised manuscript received 16 July 1998)

\begin{abstract}
Dielectric measurements were done on cosputtered metal-insulator nanocomposite films with metal volume fraction above the metal-insulator transition, in the frequency range between $20 \mathrm{~Hz}$ and $30 \mathrm{MHz}$. At intermediate and high frequency, the dielectric function can be qualitatively described in terms of the percolation theory. In the low-frequency region, we observe a sharp relaxation-type increase of the real part of dielectric constant with decreasing frequency, while the imaginary part is dominated by dc conductivity. We suggest that the low-frequency behavior may be due to a combination of metallic and tunneling conduction in the system. [S0163-1829(98)51644-X]
\end{abstract}

Granular composites consisting of nanometer-scale metal particles immersed in an insulating matrix (cermets) have attracted considerable interest due to their remarkable transport, magnetotransport, dielectric, and magnetic behavior. ${ }^{1}$ The properties of cermets can be altered in a wide range by changing the metal volume fraction $x$. They undergo an insulator to metal transition as $x$ accedes a critical value of $x_{c}$. A recent discovery of the giant Hall effect and giant magnetoresistance in cermets, ${ }^{2-7}$ where the metallic component is magnetic, intensified discussions on the dominating chargetransfer mechanisms in these systems, in particular when the concentration of metal is close to and above the critical value. Measurements of the frequency dependences of impedance or dielectric constant provide a sensitive tool for probing the charge transfer and polarization in complex systems (for a review see Ref. 8). A few examples of such studies for both nanometer-scale and micrometer-scale granular metal-insulator composites have been reported in Refs. 9-17. For composites close to the metal-insulator transition, the results are often compared to the predictions of percolation scaling theories, ${ }^{18-20}$ with the main emphasis to the high-frequency dispersion. In this interpretation, the critical volume fraction of metal $x_{c}$ is associated with the percolation threshold. ${ }^{21}$

In this paper we mainly focus on the low-frequency dielectric properties of metal-insulator nanocomposite films with the metal volume fraction $x>x_{c}$. The results are presented in terms of the effective complex capacitance. The most striking result is the rapid increase of the effective real capacitance of conducting samples at low frequency, where the apparent imaginary part of capacitance is dominated by dc conductance. We attribute this behavior to a combination of metallic conduction with frequency-dependent tunneling processes. In our simple model, the low-frequency real capacitance is associated with the tunneling correlation length $^{21,22} \xi_{t}$, which can be much greater than the classical percolation correlation length $\xi_{p}$.

Preparation and characterization of the approximately 1$\mu$ m-thick cosputtered $\left(\mathrm{Ni}_{85} \mathrm{Fe}_{15}\right)_{x}-\left(\mathrm{SiO}_{2}\right)_{1-x}$ samples on glass substrates have been described in Refs. 2, 6, and 7. The transmission electron microscopy studies show ${ }^{6,7}$ that these composites consist of crystalline metal particles with the minimum diameters down to less than $1 \mathrm{~nm}$ immersed in the amorphous insulating matrix. The values of $x$ were measured by energy dispersive $\mathrm{x}$-ray analysis. ${ }^{2}$ In the samples with $x$ $<x_{c} \approx 0.53$, the dc conduction is typical of an insulator, with the thermally activated hopping between the metal grains: $\rho \propto \exp \left[-T_{0} / T\right]^{n}$ (Ref. 1), where $T_{0}$ and $n$ are constants, $n \approx 0.4$. Following Ref. 7 , we define $x_{c}$ as the metal-insulator transition, or the percolation threshold. The results presented in this paper are for six samples with $x=0.55,0.58,0.63$, $0.64,0.65$, and 0.72 . The room temperature dc resistivity of these samples decreases from about 1 to $10^{-3} \Omega-\mathrm{cm}$ with increasing concentration. Resistivity of the samples with $x=0.55$ and 0.58 depends on temperature approximately as $\rho \propto-\log (T / \Theta)$, where $\Theta$ is a constant, up to room temperatures. The samples, with $x=0.63,0.64,0.65$, and 0.72 have small positive temperature coefficient of resistivity at room temperature, which crosses over to negative at a lower temperature, the crossover temperature being dependent on $x$. We note that there is still no unanimous understanding of both the degree of connectivity of metal grains and the dominating conduction mechanisms in samples having such properties.

The effective real $C^{\prime}$ and imaginary $C^{\prime \prime}$ parts of capacitance of rectangular samples with the length of $10-25 \mathrm{~mm}$ and width 1-2 $\mathrm{mm}$ were measured in the direction parallel to the sample plane, using a four probe method. The drive and sense contacts were well separated, to minimize the contact effects. The measurements were carried out on an HP 4284A RLC meter in the frequency range between $20 \mathrm{~Hz}$ and 1 $\mathrm{MHz}$, and HP 4285A RLC meter in the range $75 \mathrm{kHz}$ through $30 \mathrm{MHz}$, with standard "short" and "open' corrections, at room temperature. The values of the parallel capacitance and conductance $C_{p}$ and $G$, measured in the parallel equivalent circuit mode, were converted into the complex capacitance using the relations $C^{\prime}=C_{p}$ and $C^{\prime \prime}=G /(2 \pi f)$, where $f$ is the frequency. The geometry used in this work is not conventional for dielectric measurements, which are usually done perpendicular to the sample plane, with the sample placed between two capacitor plates. Measurements would have been impossible in the conventional geometry because 


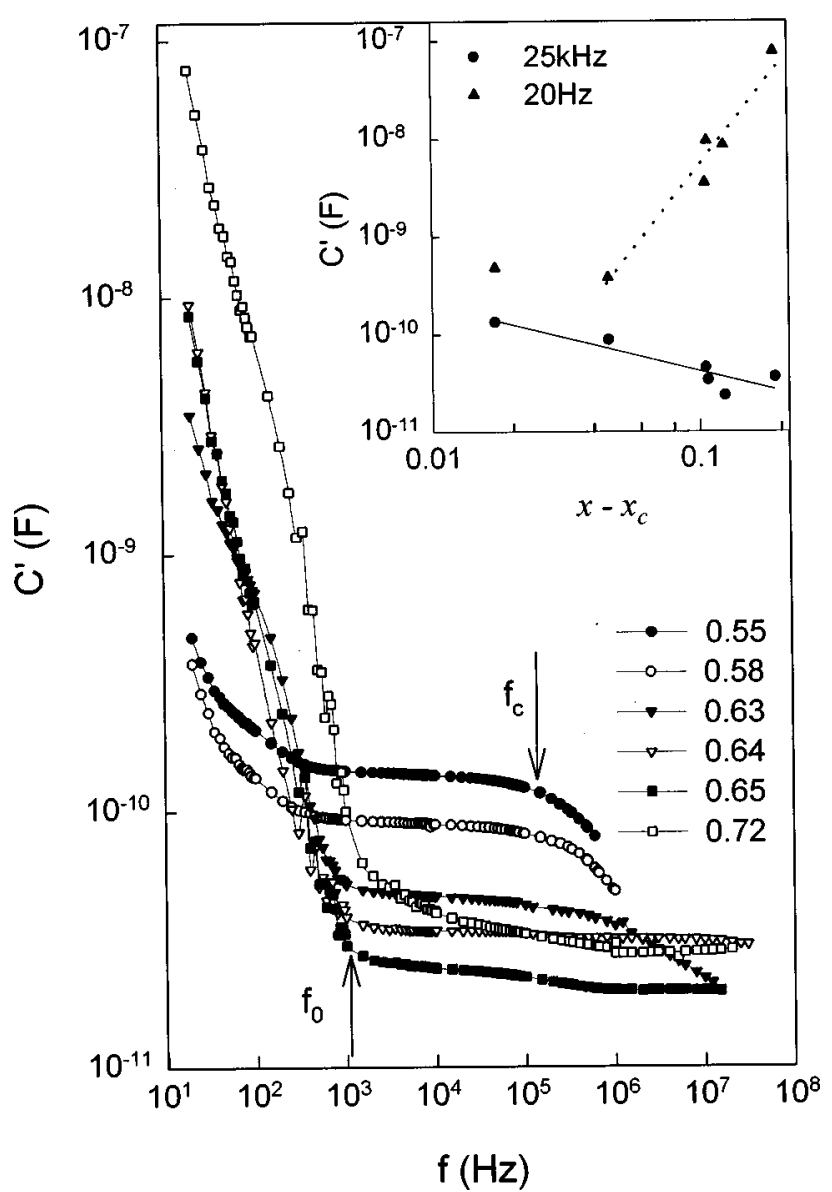

FIG. 1. Dependencies of the real part of capacitance $C^{\prime}$ on frequency for the $(\mathrm{NiFe})_{x}-\left(\mathrm{SiO}_{2}\right)_{1-x}$ samples. The metal volume fraction $x$ is shown in the figure. The inset shows a dependence of capacitance at $25 \mathrm{kHz}$ (circles) and at $20 \mathrm{~Hz}$ (triangles) on $\Delta x=x$ $-x_{c}$, on a double logarithmic scale. The solid line is the best fit to a power law for the $25-\mathrm{kHz}$ data. The dashed line is a guide for the eye.

of the relatively high conductivity of the films. In the following we assume that the measured real capacitance $C^{\prime}$ is proportional to the real part of the dielectric constant. The apparent imaginary part of capacitance $C^{\prime \prime}$ contains contributions from both the true dielectric loss and the dc conductance. The measuring instrument cannot separate these two contributions. ${ }^{23}$

Figure 1 shows the real part of capacitance $C^{\prime}$ as a function of frequency, on a double logarithmic scale, for $(\mathrm{NiFe})_{x}-\left(\mathrm{SiO}_{2}\right)_{1-x}$ samples with $x>x_{c}$. The capacitance decreases with frequency in the whole measurement range. Three different types of behavior can be noticed in Fig. 1. At frequencies greater than $f_{0} \sim 10^{2}-10^{3} \mathrm{~Hz}$, where $f_{0}$ is referred to as the cutoff frequency, but smaller than $f_{c}$, where $f_{c}$ is the crossover frequency, $C^{\prime}$ is almost constant or decreases slowly with frequency. The values of $f_{0}$ and $f_{c}$ are marked with arrows in Fig. 1. For the three samples closest to the transition, a considerable change in the slope of the curves is observed near $f_{c}$. The crossover frequency is of the order of $10^{5} \mathrm{~Hz}$ for $x=0.55$ and 0.58 ; it reaches $\sim 10^{6} \mathrm{~Hz}$ for $x=0.63$, and is out of the measurement range for other

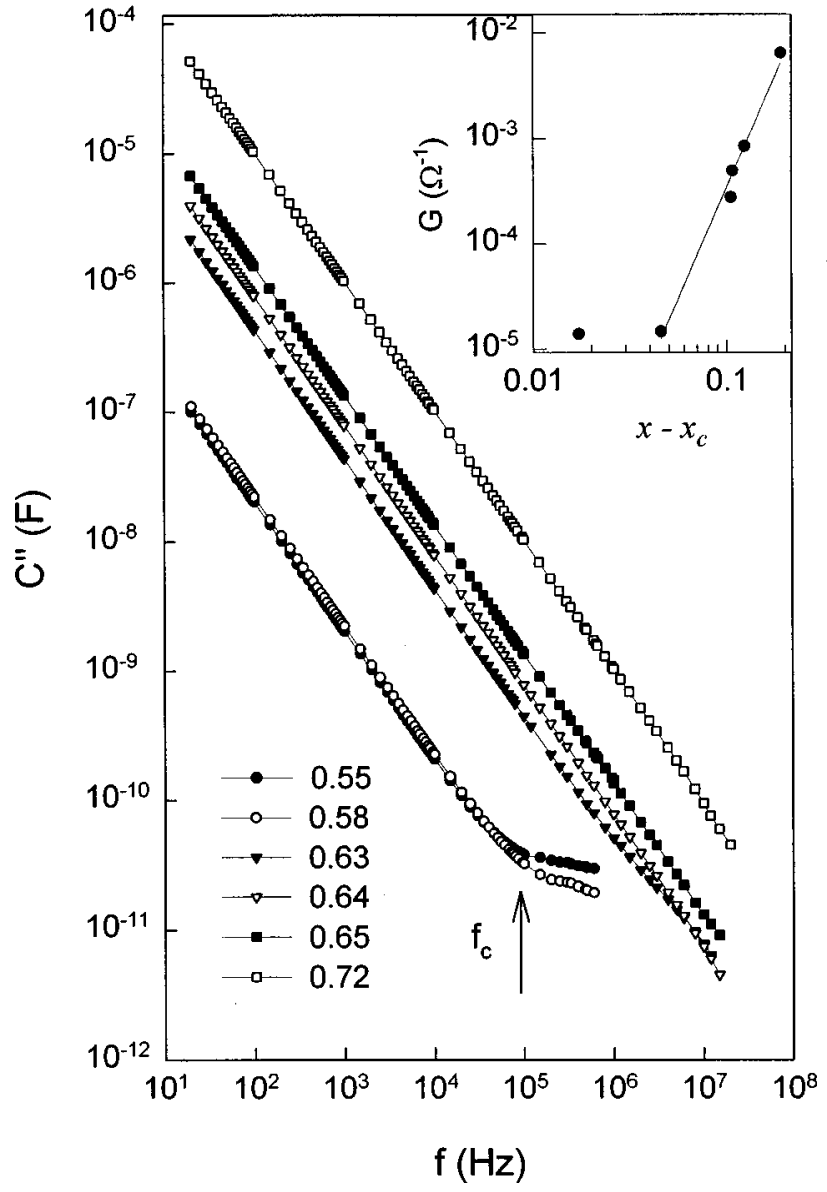

FIG. 2. Frequency dependencies of the effective imaginary part of capacitance $C^{\prime \prime}$ for the $(\mathrm{NiFe})_{x}-\left(\mathrm{SiO}_{2}\right)_{1-x}$ samples. The inset shows a dependence of $G$ at $25 \mathrm{kHz}$ on $\Delta x=x-x_{c}$, on a double logarithmic scale, where the solid line is the best fit to a power law.

samples. We approximated the behavior of the real capacitance at $f>f_{c}$ with a power-law dependence of the form $C^{\prime} \sim f^{-y}$. The estimations of the critical exponent $y$ give the values of $y \approx 0.21$ (at $f \sim 10^{6} \mathrm{~Hz}$ for $x=0.55$ ), $y \approx 0.41$ (at $f$ $\sim 10^{6} \mathrm{~Hz}$ for $x=0.58$ ), and $y \approx 0.23$ (at $f \sim 10^{7} \mathrm{~Hz}$ for $x=0.63$ ). The cutoff frequency $f_{0}$ is of the order of $10^{2} \mathrm{~Hz}$ for two samples closest to the percolation threshold, and increases to over $10^{3} \mathrm{~Hz}$ when $x=0.72$. At low frequencies, $f<f_{0}$, the value of $C^{\prime}$ increases sharply with decreasing frequency. One can also notice a kink in the dependence at a lower frequency.

The dependence of the real capacitance $C^{\prime}$ on the value of $\Delta x=x-x_{c}$ on a double logarithmic scale is shown in the inset of Fig. 1. At intermediate frequency of $25 \mathrm{kHz}$ (filled circles) a power-law dependence of the form $C^{\prime} \propto(\Delta x)^{-s}$ is observed. The solid line in the plot shows the best fit to this dependence with $s=0.7 \pm 0.1$. In contrast, the effective capacitance measured at a low frequency $f=20 \mathrm{~Hz}$ (shown by filled triangles) increases with $x$. To the best of our knowledge, the low-frequency behavior of capacitance of conducting metal-insulator composite samples, such as shown in Fig. 1 , has not been reported before.

The frequency dependence of the imaginary part of capacitance $C^{\prime \prime}$ for the same set of samples is shown in Fig. 2. 
At low and intermediate frequencies, $f<f_{c}$, the values of $C^{\prime \prime}$ are inverse proportional to frequency within the experimental accuracy, $C^{\prime \prime} \propto 1 / f$. This is an indication of the dominant contribution of dc conductance. ${ }^{23}$ For the samples closest to percolation, a dispersion of the form $C^{\prime \prime} \propto f^{-y}$ is observed at high frequency $f>f_{c}$, where $y \approx 0.14,0.33$, and 0.82 , respectively, for samples with $x=0.55,0.58$, and 0.63 . In the latter case, the dispersion may not be well developed. The dependence of the real conductance $G=2 \pi f C^{\prime \prime}$ on $\Delta x$, at $f=25$ $\mathrm{kHz}$, is shown in the inset of Fig. 2 on a double logarithmic scale. The conductance can be represented as a power-law function of metal volume fraction $G \propto \Delta x{ }^{t}$. However, we notice that this dependence does not extend to the closest vicinity of the percolation threshold. The critical exponent $t$ has a value of $t \approx 4.2$, which is a little lower than that found in Ref. 7 for the same samples in low-temperature dc measurements. We carried out measurements on several cosputtered $\mathrm{Pt}-\mathrm{SiO}_{2}$ and $\mathrm{Au}-\mathrm{SiO}_{2}$ samples, where the dielectric behavior was found qualitatively similar to that of $(\mathrm{NiFe})-\left(\mathrm{SiO}_{2}\right)$ films.

The intermediate- and high-frequency behavior of the complex capacitance can be qualitatively understood as due to percolation-type scaling. In the percolation models, for frequencies much smaller than the plasmon frequency of metal, the composite system is considered as a random mixture of metallic regions with real conductivity $\sigma_{m}$ and dielectric regions with real dielectric constant $\varepsilon_{d}$. The effective dc conductivity of the percolation system $\sigma(0)$ is determined by the infinite percolation cluster above the percolation transition, while the effective dielectric constant $\varepsilon(0)$ arises from charge polarization in the finite metallic clusters of the typical size $\xi_{p}$. The diverging percolation correlation length $\xi_{p} \propto|\Delta x|^{-\nu}$, where $x$ is the concentration of metal, $\Delta x=x-x_{c}$ and $\nu \approx 0.9$ in $3 d,{ }^{24}$ is the scale of inhomogeneity of the percolation problem. ${ }^{21}$ The static conductivity and dielectric constant are described as power functions of $\Delta x$, namely, $\sigma(0) \propto(\Delta x)^{t}$ (for $x>x_{c}$ ) and $\varepsilon(0) \propto|\Delta x|^{-s}$, where $t$ and $s$ are the critical exponents. For a 3-dimensional array, in the simplest approximation, $\sigma(0)$ and $\varepsilon(0)$ scale with $\xi_{p}$ as $\sigma(0) \propto \xi_{p}^{-2}$ (for $\left.x>x_{c}\right), \varepsilon(0) \propto \xi_{p}$. This gives a simple estimate for the values of the critical exponents $t \approx 1.8$, and $s \approx 0.9$, which are not too different from the elaborate computer simulation results: $t \approx 2.0$ and $s \approx 0.76 .{ }^{24}$ We note that the experimental dependence of the real capacitance $C^{\prime}$ at $f=25 \mathrm{~Hz}$ on concentration, shown in the inset of Fig. 1, is in agreement with the theoretical result, the experimental value of $s$ being $s=0.7 \pm 0.1$. While the real effective dc conductance shows a nonuniversal behavior with $t \approx 4.2$, the nonuniversal values of $t$ were observed in cermets before and are sometimes attributed to tunneling effects. ${ }^{25}$

Two well-known variations of the classical scaling theory of frequency dispersion close to the percolation transition, $|\Delta x| \ll 1$, are based on the intercluster polarization approach, ${ }^{18,19}$ or on anomalous diffusion on noninteracting fractal clusters. ${ }^{20}$ The frequency dependence of the complex effective conductivity $\sigma(\omega)$ and dielectric constant $\varepsilon(\omega)$ is determined by the value of the scaling parameter $z$ $=h / \Delta x^{s+t}$, where $h=\left(\omega \varepsilon_{0} \varepsilon_{d}\right) / \sigma_{m}$ is the "conductivity ratio,' $\omega=2 \pi f$, and $\varepsilon_{0}$ is the permittivity of free space. When the value of $z$ is small, $z \ll 1$, and $\Delta x>0$, the real part of dielectric constant is constant $\varepsilon^{\prime}(\omega)=$ const, and the imaginary part is inverse proportional to frequency, $\varepsilon^{\prime \prime}(\omega)$ $\propto \omega^{-1}$. The latter equation is the signature of dominant contribution of conduction via the infinite cluster. When $z \gg 1$, the dielectric constant follows a power-law dependence on frequency, $\varepsilon(\omega) \propto \omega^{-y}$, where the critical exponent $y=s /(s$ $+t) \approx 0.27$, using the universal values of $s$ and $t$. This dispersive behavior can be observed when the frequency is greater than the crossover value, which can be estimated from the condition $z \cong 1$, or $\omega_{c}=2 \pi f_{c} \cong \Delta x^{s+t} \sigma_{m} /\left(\varepsilon_{0} / \varepsilon_{d}\right)$. It can be easily checked that this estimate exceeds considerably the values of $f_{c}$ that have been observed in many experiments. $^{10-17}$ In our experiments (Figs. 1 and 2) the crossover frequency is also much lower that this estimate. The theoretical value of $y \approx 0.27$ can be compared to the experimental results discussed above. The experiment is qualitatively in line with the theory. However, the values of the exponent deviate from the predictions, in particular for the sample with $x=0.63$, where the value of $y$ about 0.23 is found based on the $C^{\prime}$ data, and is 0.82 based on the $C^{\prime \prime}$ data. A relatively narrow frequency range for the evaluation of the exponents may be one reason for these discrepancies.

The sharp increase of the effective capacitance at low frequency (Fig. 1) cannot be explained by the percolation theory. We consider tunneling as a possible reason. The percolation problem with tunneling for metal-insulator composites below the percolation threshold has been considered both for dc conductivity, ${ }^{25}$ and ac conductivity. ${ }^{22}$ We suggest that both the metallic spanning cluster and tunneling junctions have to be taken into account for ac properties of conducting $\left(x>x_{c}\right)$ nanocomposite films. Qualitatively, it can be done as follows. Let us consider the system as consisting of two independent components. The first, metallic subsystem, consists of the infinite percolation cluster and the finite clusters of metal particles discussed above. Its properties can be described by the classical percolation theory. The second component is composed of tunneling junctions between the finite clusters. The typical tunneling distances $r$ may be distributed between $d$ and $\xi_{p}$, where $d$ is the particle size. Neglecting the charging effects, the probability of phononassisted tunneling between metal clusters in unit time, or tunneling frequency, is ${ }^{26} \tau^{-1} f_{p h} \exp [-2 r / a]$, where $f_{p h}$ $\sim 10^{12} \mathrm{~Hz}$ is the typical phonon frequency and $a$ of the order of several $\AA$ is the localization length. As $r$ is in general much greater than $a$, the resistivity of the tunneling subsystem is much greater than that of the metal infinite cluster. Following Ref. 22, we can consider each finite cluster of size $\sim \xi_{p}$ as a node for a new percolation problem where the resistance between adjacent nodes is $R_{i j}=R_{0} \exp [\lambda]$. In our case, the values of $\lambda$ are distributed between $2 d / a$ and $2 \tau_{p} / a$. For the system with the exponentially wide distribution of resistances, ${ }^{21}$ only a small fraction of tunneling junctions determine conductivity. These important junctions form a network with a typical separation $\xi_{t}$, or the tunneling correlation length, which may be much greater than the percolation correlation length. A simple estimate ${ }^{21}$ for this value gives $\xi_{t} \sim \xi_{p} \lambda_{m}^{\nu} \sim \xi_{p}^{\nu+1} / a^{\nu}$, where $\lambda_{m}$ is the maximum value of the random parameter $\lambda$. As noted in Refs. 21 and 22, $\xi_{t}$ is the true scale of inhomogeneity of field and current for the problem with tunneling, from which we assume that both the effective conductivity and dielectric constant of the tunneling 
subsystem should scale with $\xi_{t}$, in particular, $\varepsilon_{t} \propto \xi_{t}$. Tunneling requires a wait time $\tau \propto\left(f_{\mathrm{ph}}\right)^{-1} \exp [2 r / a]$. This process is thus released only at low frequency $f \ll 1 / \tau$. This is why the sharp increase of the effective capacitance is observed only at low frequency (Fig. 1). One can estimate the cutoff frequency as $f_{c} \sim f_{\mathrm{ph}} \mathrm{exp}[-2 d / a]$. Assuming $a \sim 2 \AA$, the cutoff frequency of $10^{3} \mathrm{~Hz}$ (Fig. 1) would correspond to a typical tunneling distance about $20 \AA$. Thus the relaxation-type ${ }^{23}$ behavior below the cutoff frequency $f_{0}$ corresponds to tunneling by a distance of the order of particle diameter.

For an intuitive understanding of why only the real part of capacitance, but not conductance, is dominated by tunneling at low frequency, we can suggest a qualitative equivalent $R-C$ circuit of the system, where the innercluster tunneling component with parameters $R_{t}$ and $C_{t}$ is in parallel with the metal component of $R_{m}$ and $C_{m}$. At low frequency, $R_{t}$ $\gg R_{m}$ and $C_{t} \gg C_{m}$. Then capacitance is dominated by tunneling, and resistance is dominated by the metal channels.
To conclude, the dielectric measurements on $\mathrm{NiFe}-\mathrm{SiO}_{2}, \mathrm{Pt}-\mathrm{SiO}_{2}$ and $\mathrm{Au}-\mathrm{SiO}_{2}$ granular cosputtered films close to the metal-insulator transition and in the metallic state suggest that tunneling dominates the real part of lowfrequency effective dielectric constant. We associate it with the frequency-dependent tunneling correlation length. A percolation-type behavior is observed at intermediate and high frequency. The behavior of the effective capacitance at frequencies lower than our measurement range would be an interesting experimental problem. The effective dielectric constant should be finite at $f \rightarrow 0$. We thus expect more interesting features at low frequency.

We wish to thank G. A. Niklasson and Z.-Q. Zhang for helpful comments on this paper. This work was supported by Hong Kong RGC Grant Nos. HKUST 6129/97P and (HKUST) DAG 97/98. SC27.
*Electronic address: phalec@usthk.ust.hk or alec@mhd.ioffe.rssi.ru

${ }^{1}$ B. Abeles, Appl. Solid State Sci. 6, 1 (1976); G. A. Niklasson and C. G. Granqvist, J. Appl. Phys. 55, 3382 (1984); Ping Sheng, Philos. Mag. B 65, 357 (1992).

${ }^{2}$ A. B. Pakhomov, X. Yan, and B. Zhao, Appl. Phys. Lett. 67, 3497 (1995); A. B. Pakhomov, X. Yan, and Y. Xu, J. Appl. Phys. 79, 6140 (1996).

${ }^{3}$ A. Milner, A. Gerber, B. Groisman, M. Karpovsky, and A. Gladkikh, Phys. Rev. Lett. 76, 475 (1996).

${ }^{4}$ B. Zhao, X. Yan, and A. B. Pakhomov, J. Appl. Phys. 81, 5527 (1997).

${ }^{5}$ B. A. Aronzon, A. A. Likalter, V. V. Rylkov, A. K. Sarychev, M. A. Sedova, and A. E. Varfolomeev, Phys. Status Solidi B 205, 151 (1998).

${ }^{6}$ X. N. Jing, N. Wang, A. B. Pakhomov, K. K. Fung, and X. Yan, Phys. Rev. B 53, 14032 (1996).

${ }^{7}$ A. B. Pakhomov, X. Yan, N. Wang, X. N. Jing, B. Zhao, K. K. Fung, J. Xhie, T. F. Hung, and S. K. Wong, Physica A 241, 344 (1997).

${ }^{8}$ G. A. Niklasson, J. Appl. Phys. 62, R1 (1987).

${ }^{9}$ R. M. Hill, Thin Solid Films 125, 277 (1985).

${ }^{10}$ L. Benguigui, J. Phys. (France) Lett. 46, L1015 (1985).

${ }^{11}$ R. B. Laibowitz and Y. Gefen, Phys. Rev. Lett. 53, 380 (1984).

${ }^{12}$ M. F. Hundley and A. Zettl, Phys. Rev. B 38, 10290 (1988).

${ }^{13}$ Y. Song, Tae Woh Noh, Sung-ik Lee, and J. R. Gaines, Phys. Rev. B 33, 904 (1986).

${ }^{14}$ D. S. McLachlan, I. I. Oblakova, and A. B. Pakhomov, Physica A 207, 234 (1994).
${ }^{15}$ D. S. McLachlan, A. B. Pakhomov, I. I. Oblakova, F. Brouers, and A. K. Sarychev, in Electrically Based Microstructural Characterization, edited by R. A. Gerhadt, S. R. Taylor, and E. J. Garboczi, MRS Symposia Proceedings No. 411 (Materials Research Society, Pittsburgh, 1996), p. 333.

${ }^{16}$ F. Brouers, I. Royen, S. Blacher, R. Pirard, F. Gubbels, R. Jerome, and A. Sarychev, in Electrically Based Microstructural Characterization (Ref. 15), p. 339.

${ }^{17}$ K. Brantervik and G. A. Niklasson, Thin Solid Films 165, 67 (1988).

${ }^{18}$ A. L. Efros and B. I. Shklovskii, Phys. Status Solidi B 76, 474 (1976).

${ }^{19}$ D. J. Bergman and Y. Imry, Phys. Rev. Lett. 39, 1222 (1977).

${ }^{20}$ Y. Gefen, A. Aharony, and S. Alexander, Phys. Rev. Lett. 50, 77 (1983).

${ }^{21}$ B. I. Shklovskii and A. L. Efros, Electronic Properties of Doped Semiconductors (Springer-Verlag, Berlin, 1994).

${ }^{22}$ A. K. Sarychev and F. Brouers, Phys. Rev. Lett. 73, 2895 (1994).

${ }^{23}$ A. K. Jonscher, Dielectric Relaxation in Solids (Chelsea Dielectric Press, London, 1983).

${ }^{24}$ D. J. Bergman and D. Stroud, Solid State Phys. 46, 146 (1992).

${ }^{25}$ I. Balberg, N. Wagner, Y. Goldstain, and S. Z. Weisz, in Physical Phenomena in Granular Materials, edited by G. D. Cody, T. H. Geballe, and P. Sheng, MRS Symposia Proceedings, No. 195 (Materials Research Society, Pittsburgh, 1990), p. 333.

${ }^{26}$ A. L. Efros, Philos. Mag. B 43, 829 (1981). 